\title{
Vascular Access-Related Infections in HIV Patients Undergoing Hemodialysis: Case Description and Literature Review
}

\author{
Carlos E. Figueroa Castro ${ }^{1}$ and Miguel G. Madariaga ${ }^{2}$ \\ ${ }^{1}$ Instructor in Medical Microbiology and Medicine, Creighton University School of Medicine, Department of Medicine, Division of Infectious \\ Diseases; ${ }^{2}$ Assistant Professor, University of Nebraska Medical Center, Department of Medicine, Division of Infectious Diseases; Omaha, NE, USA
}

\begin{abstract}
Poor immune status, the use of a vascular access different from an AV fistula, and intravenous drug use (IDU) may favor increased rates of vascular access infections among HIV infected patients on hemodialysis. Staphylococcus spp. and Streptococcus spp. are the main cause of these infections, but Gram-negative rods and fungi have been found as well. Using an AV fistula when possible, and eliciting a history of IVDU on every visit may prevent this type of infection. When infections are present, coverage for both Gram-positive and negative organisms is recommended. Additional studies specifically addressing the issue of vascular access infection in HIV infected patients are required. Key-Words: HIV, chronic kidney disease, renal dialysis, catheter-related infections.
\end{abstract}

Chronic kidney disease (CKD) is a common finding in HIVinfected patients. Renal replacement therapy with hemodialysis is required in a proportion of them, especially when HIV-associated nephropathy (HIVAN) is present. Vascular access-related infections are a known complication of hemodialysis. In this article, we presented an illustrative case, and we performed a literature search regarding vascular access-related infections among HIV patients undergoing hemodialysis. We searched the Cochrane Central Register of Controlled Trials and MEDLINE, including articles in English language from January 1981 to April 2008. We also searched the reference lists of selected articles. Randomized controlled trials were not found. Observational studies in vascular-access related infections in HIV patients were selected. We found eight studies, (HIV-positive patients $=311$ ). We reviewed the effect of HIV infection in the development of vascular access infection, the effect of the type of access in the development of infection, the microbiology of the vascular infections, and other risk factors for the development of infection.

\section{Case Report}

A 34 year-old African American male with HIV-1 infection for 12 years and end-stage renal disease secondary to HIVassociated nephropathy (HIVAN) on hemodialysis for the last six months was admitted for further evaluation of a positive blood culture for methicillin-resistant Staphylococcus aureus (MRSA) obtained during his dialysis session. He complained of fever $\left(103^{\circ} \mathrm{F}\right)$, chills, chest pain, and purulent discharge from his left groin arteriovenous (AV) graft. His past medical history was also relevant for Moraxella catarrhalis pneumonia, bilateral saphenous vein thrombosis, and seizure disorder. The patient's medications included lopinavir/ ritonavir, emtricitabine, and tenofovir, in renally adjusted

Received on 19 August 2008; revised 26 November 2008.

Address for correspondence: Dr. Carlos E. Figueroa Castro, MD, Creighton University School of Medicine, 4101 Woolworth Avenue 111D, Omaha, NE 68105. Phone: (402) 995-5219, Fax: (402) 9775606. E-mail: carlos_figueroa_c@yahoo.com.

The Brazilian Journal of Infectious Diseases 2008;12(6):531-535. (C) 2008 by The Brazilian Journal of Infectious Diseases and Contexto Publishing. All rights reserved. doses, in addition to monthly inhaled pentamidine for prevention of Pneumocystis jirovecii pneumonia, aspirin, erythropoietin, calcium carbonate, iron sulfate, and valproic acid. At admission, he required intensive care unit monitoring due to significant hypotension (blood pressure: 70/40 mm $\mathrm{Hg})$. He was initially treated empirically with vancomycin, levofloxacin, and gentamicin, in addition to aggressive fluid resuscitation. When the blood cultures drawn on admission and from the AV graft revealed MRSA (susceptible to minocycline,trimethoprim/sulfamethoxazole, and vancomycin), his antibiotics were simplified to vancomycin. Repeated recovery of MRSA from blood cultures despite adequate antimicrobial therapy raised suspicion for deep AV graft infection. The access was surgically removed, after significant amount of purulent material in the tunnel graft and the arterial anastomosis was found. A transthoracic echocardiogram showed a depressed left ventricular function, but no evidence of vegetations, or myocardial abscesses. After the removal of the infected AV graft, repeated blood cultures became negative. He received four weeks of vancomycin and he continues to receive hemodialysis through a permanent central venous dialysis catheter, awaiting for the placement of a new dialysis access.

\section{Background}

Since 1981, human immunodeficiency virus (HIV) infection has affected more than a million people in the United States [1], with an estimated 40.000 newly infected individuals every year [2]. Kidney disease was described early in the HIV epidemic [3]. It is particularly common among patients not receiving antiretroviral therapy, affecting up to $30 \%$ of HIV patients. Kidney disease is associated with progression to AIDS and death [4]. The etiologies of chronic kidney disease in HIV-infected individuals are multiple (Table 1). However, the most common cause of end-stage renal disease in HIVinfected individuals undergoing hemodialysis is HIVassociated nephropathy (HIVAN), which is a collapsing form of focal segmental glomerulosclerosis [3,4]. HIV-infected individuals may require renal replacement therapy [4].

The methods of renal replacement therapy in HIV-infected individuals with kidney disease include, as in the general 
population, hemodialysis or peritoneal dialysis. In 2000, almost $2 \%$ of dialysis patients were reported to have either HIV or AIDS [4]. However, the true incidence and prevalence may be higher due to incomplete reporting and heterogenous case distribution. For example, HIV-infected individuals constituted up to $40 \%$ of dialysis patients in urban centers in the preHAART era [5]. Based on a 2002 study on survival among HIV-infected individuals using the United States Renal Data System (USRDS), a national database system that collects, analyzes, and distributes information about end-stage renal disease in the United States, 6179 individuals with HIVAN or HIV infection on dialysis were identified [6]. USRDS comprises more than $90 \%$ of the individuals requiring hemodialysis in the United States [7]. African Americans account for the majority of patients with HIV infection undergoing hemodialysis [2,4]. Antiretroviral therapy has shown survival benefits in HIV individuals [8], including patient undergoing hemodialysis; however, the overall survival is still lower than HIV-negative individuals on dialysis [6]. Since diabetes and cardiovascular disease are increasing among the HIV-infected population [9], a higher number of these patients suffering from chronic kidney disease, including end-stage renal disease, is expected.

Hemodialysis requires a vascular access for the removal of toxic products. There are three types of vascular access for hemodialysis: arteriovenous fistula, arteriovenous graft, and tunneled central venous access. Vascular access-related infection is a known complication of hemodialysis. These infections are associated with significant morbidity and mortality not only directly related to the infectious process itself, but also from the medical and surgical procedures required to treat this infections. Known risk factors for the development of vascular-access related infections are diabetes, low albumin level, and especially, the type of vascular access [10]. Because of the lower rate of vascular accessrelated complications with the use of arteriovenous fistula (including infectious complications) when compared with other methods of vascular access, the National Kidney Foundation Diabetes Outcome Quality Initiative has recommended the use of this type of access when possible [11]. This recommendation has been extended to HIV individuals undergoing hemodialysis [4,12,13].

In order to review the effect of HIV infection in the development of vascular access-related infection on HIVinfected patients undergoing hemodialysis, we performed a systematic literature search for this review. We searched the Cochrane Central Register of Controlled Trials and MEDLINE, including articles only in English language from January 1981 to April 2008. We used the terms "HIV", "hemodialysis", and "infection". Reference lists of retrieved articles were reviewed. We reviewed articles that described rates of infection based on HIV status and type of access. Eight observational studies were included in this review [14-21], because of their emphasis in infection, rather than other complications like occlusion or thrombosis. We did not find randomized controlled trials. The total number of HIV-positive patients studied was 311. The characteristics of the studies retrieved are shown on Table 2 .

\section{Results}

The majority of the studies included in this review were performed during or after the introduction of highly active antiretroviral therapy. The effect of HIV status on hemodialysis vascular access infections is difficult to generalize, due to the small number of patient included in these studies. Based on the presence or absence of HIV infection (without distinction based on immune status such as CD4+ counts, or presence of opportunistic infections), different conclusions were found. Some studies claim no differences between HIV positive and negative patients $[14,17,18,20]$. Nannery et al. [14] described infection rates $(17 \%)$ that were similar to historical rates $(6 \%$ 25\%) at the time of publication. Mokrzycki et al. [17] did not find differences in the rate of bacteremia, exit site infections, or the need for catheter removal among patients with and without HIV infection. Mitchell et al. [20] did not find statistically significant differences in the infection-free time among patient with tunneled cuffed catheters, regardless of their HIV status.

In contrast, other studies have found differences between HIV positive and negative patients [15,16,21]. Brock et al. [15] described a significant difference in the infection rates between HIV positive and negative patients undergoing hemodialysis (40\% vs. 14\%). A more striking difference was found by Curi et al.: 24\% vs. 3\% (16). Another article by Mitchell et al. [21] found a lower infection-free graft survival among HIV patients (hazard ratio $=3.51,95 \%$ CI: 1.21-18.85, $\mathrm{p}=0.025$ ). This study also suggested that HIV status is an important risk factor for infection, when an AF graft is chosen as the vascular access for hemodialysis, as compared with AV fistulas.

The majority of studies made statistical analysis based on patient's immune status [14-17,19,21]. The results have not been uniform either. Nannery et al. [14] distinguished between HIV and AIDS patients (based on the 1987 CDC surveillance definition). The rates of infection were $40 \%$ for the AIDS group vs. $18 \%$ for the HIV+ group (although the total number of infections was four). Brock et al. [15] found a statistically significant difference between HIV+ and AIDS (defined as presence of opportunistic infections), but interestingly, the overall higher rate of infections happened in the HIV+ group (44\% vs. 27\%). A second analysis involving only AV graft infections was performed, This revealed an increased rate of infection based on the patient's immune status (HIV-: 15\%, HIV+: 36\%, AIDS: 43\%, p<0.05). Mokrzycki et al. [17] found an inverse relation between CD4 count and antibiotic prophylaxis (trimethoprim-sulfamethoxazole, fluconazole, clarythromycin) and the rate of exit site infection, but not bacteremic episodes, in HIV patients with history of intravenous drug use (IDU). For non-IDU patients, only the CD4 count was inversely associated with exit site infection.

Other studies based on immune status of patients have showed different results as mentioned above. Curi et al. [16] 
Table 1. Conditions associated with chronic kidney disease among HIV-infected patients.

HIV-associated nephropathy (HIVAN)

Membranoproliferative glomerulonephritis

Mesangial glomerulonephritis with immune deposits

Minimal change disease

Lupus-like glomerulonephritis

Crystal-induced nephropathy

Fanconi syndrome

Allergic interstitial nephritis

Thrombotic thrombocytopenic purpura/Hemolytic uremic syndrome

Paraproteinemia

Lymphoma

Table 2. Description of studies included in this review.

\begin{tabular}{|c|c|c|c|c|c|c|}
\hline Investigator & $\begin{array}{l}\text { Study l } \\
\text { location }\end{array}$ & $\begin{array}{c}\text { Number of } \\
\text { enrolled patients } \\
\text { (HIV Patients) }\end{array}$ & $\begin{array}{c}\text { All } \\
\text { infections }\end{array}$ & $\begin{array}{l}\text { AV fistula } \\
\text { infections }\end{array}$ & $\begin{array}{l}\text { AV graft } \\
\text { infections }\end{array}$ & $\begin{array}{l}\text { Catheter } \\
\text { infections }\end{array}$ \\
\hline Nannery W.M., et al., 1991 [14] & Trenton, NJ & $22(22)$ & $4 / 22$ & $0 / 22$ & $4 / 18$ & $\mathrm{~N} / \mathrm{A}$ \\
\hline Brock J.S., et al., 1992 [15] & New York, NY & $108(29)$ & $11 / 29$ & $0 / 29$ & $11 / 29$ & N/A \\
\hline Curi M.A., et al., 1999 [16] & Newark, NJ & $104(42)$ & $10 / 42$ & $2 / 42$ & $8 / 42$ & N/A \\
\hline Mokrzycki M.H., et al., 2000 [17] & Bronx, NY & $81(40)$ & & & & $\begin{array}{l}2.23 \pm 0.7^{\mathrm{a}} \\
2.20 \pm 0.6^{\mathrm{b}}\end{array}$ \\
\hline Gorski T.F., et al., 2002 [18] & Brooklyn, NY & 37 (37) & $1 / 37$ & $0 / 23$ & $1 / 14$ & N/A \\
\hline Eustace J.A., et al., 2005 [19] & Baltimore, MD & $60(60)$ & $32 \%$ & $0 \%$ & $31.6 \%$ & $60.5 \%$ \\
\hline Mitchell D., et al., 2006 [20] & Birmingham, AL & 88 (33) & 17/33 & N/A & N/A & 17/33 \\
\hline Mitchell D., et al., 2007 [21] & Birmingham, AL & $62(48)$ & $6 / 48$ & $0 / 23$ & $6 / 15$ & N/A \\
\hline
\end{tabular}

a. Infection rates/1000 tunneled cuffed catheter days, bacteremia events; b. Infection rates/1000 tunneled cuffed catheter days, exit site infection events.

Table 3. Rates of AV graft infection in HIV and non-HIV infected patients undergoing hemodialysis.

\begin{tabular}{lcc}
\hline Investigator & HIV patients & Non-HIV patients \\
\hline Brock J.S., et al., 1992(15) & AIDS: 43\% & $15 \%$ \\
& HIV+: 36\% & $7 \%$ \\
Curi M.A., et al., 1999(16) & $30 \%$ & $17 \%$ \\
*Mitchell D., et al., 2007(21) & $40 \%$ & \\
\hline
\end{tabular}

* Although the infection rates were different, statistical significances was not reached $(\mathrm{p}=0.09)$. However, the infectionfree graft survival was significantly lower in the HIV positive group ( $\mathrm{p}=0.0025$ by the Log rank test).

Table 4. Organisms described as etiologic agents of vascular access infections in patients with HIV receiving hemodialysis.

\begin{tabular}{ll}
\hline Investigator & Isolates \\
\hline Nannery W.M., et al., 1991 [14] & S.aureus, coagulase-negative Staphylococci \\
Brock J.S., et al., 1992 (15] & Staphylococci, Streptococci, other (not described) \\
Mokrzycki M.H., et al., 2000 [17] & S.aureus, S.epidermidis, S.xylosus, Viridans Streptococci, A.calcoaceticus, E.cloacae, E.coli, \\
& F.indologenes, P.aeruginosa, C.albicans, C.neoformans \\
Mitchell D., et al., 2006 [20] & S.aureus, S.epidermidis, S.microaerophilus, E.cloacae, P.mirabilis, C.freundii \\
\hline
\end{tabular}


did not find differences in prosthetic and autologous vascular infection rates based on the CD4 count. Eustace et al. [19] mentioned a statistically significant association between access removal and CD4 count $(\mathrm{p}=0.02)$, but the $95 \% \mathrm{CI}$ suggested against such a difference (0.995-1.000). Mitchell et al. [21] described similar CD4 counts for patients with and without infection. The use of AV grafts among HIV-infected patients is definitively associated with higher degrees of infection, when compared with the non-HIV population, as described in Table 3. None of the studies mentioned differences in the clinical presentation of infected vascular access, but a tendency for higher rates of hospitalization was noted $[17,20]$. The majority of studies mentioned differences in occlusion rates, related mainly to the use of AV grafts $[15,16,18,19]$. Potential explanations of why HIV patients may be more prone to access infections include IDU [15-17] and hepatitis B surface antigenemia [17]. IDU is associated with the development of bacteremia. Methods involved in vascular access infection include direct inoculation of bacteria into the vascular access (when the access is used as drug delivery system), and secondary seeding in the vascular access. Hepatitis B surface antigenemia may be a marker of IDU, rather than a cause of increased vascular access infection.

Four studies [14,15,17,20] included information about the microorganisms causing vascular access infections in this population. The results are summarized in Table 4. The most detailed information regarding the microbiology of these infections was described by Mokrzycki et al. [17], and Mitchell et al. [20]. The former described a predominance of Grampositive isolates (60\%), particularly S.aureus (60\% of Grampositive organisms). Gram-negative organisms were isolated four times more frequently than in the control group of nonHIV infected patients (22\% vs. 5\%), with E.coli being the most common isolate (two out of seven). P.aeruginosa was isolated in one occasion. Fungal isolates were recovered only in the HIV positive group, with C.albicans as the most common isolate (75\%). In the other hand, Mitchell et al. [20] found a predominance of Gram-positive isolates, but the most common organism was S.epidermidis (66\% of all Gram-positive organisms). The most common Gram-negative isolate was E.cloacae (two out of three isolates). Also, there were more mixed infections in the HIV positive group, most of them caused by two Gram-positive organisms. None of the studies mentioned antimicrobial susceptibility or isolation of multidrug resistant organisms.

\section{Discussion}

Patients with HIV infection are at risk of developing kidney disease. Some of these patients may require hemodialysis as renal replacement therapy. Most of the recommendations for the management of these patients are based on studies performed in non-HIV patients.

We have reviewed the available literature concerning the management of vascular access-related infections in HIV patients undergoing hemodialysis. From the information analyzed, we could offer some recommendations for the prevention and management of this condition.

1. When possible, an AV fistula should be the preferred vascular access for hemodialysis. This recommendation is in concordance with the Infectious Diseases Society of America guideline in the management of chronic kidney disease in HIV patients, and others [4,12,13]. However, it requires validation from further studies, preferably using data from the USRDS.

2. The empirical antimicrobial treatment of these infections should include coverage for both Gram-positive cocci and Gram-negative bacilli. Initial empirical antifungal medications are probably not required. Because of the lack of information regarding antimicrobial susceptibility, physicians should have knowledge of local antimicrobial susceptibility patterns to guide their antibiotic choice. Since the rate of infection and colonization with methicillinresistant S.aureus (MRSA) in the community has increased dramatically [22-24], a combination including a glycopeptide and a $\beta$-lactam or a fluoroquinolone with Gram-negative activity seems to be a reasonable empirical choice.

3. There is insufficient evidence to suggest the routine use of antimicrobial prophylaxis for all HIV patients undergoing hemodialysis, as only one study noted a decline in exit site infections with their use [17].

4. A high index of suspicion for vascular access-related infections among HIV-infected patients is required, particularly among those with poor immune status, history of IDU, and use of AV fistula. The clinical presentation among these patients has not been well described, although it is assumed to be similar to non-HIV infected patients. An early diagnosis might translate in decreased morbidity and decreased hospitalization time. We do not have elements to recommend a more or less aggressive surgical treatment different from the non-HIV infected individual.

\section{Conclusions}

Vascular access-related infections are an important medical problem in the management of patients with HIV undergoing hemodialysis. The studies in this area suggest the benefits of using an AV fistula as the preferred vascular access method; however, the small size of these studies limits the strength of this conclusion. The most common organism involved in these infections are Grampositive organisms like staphylococci and streptococci, but Gram-negative bacilli and fungi have been described as well. The clinical manifestations do not seem to differ from non-HIV-infected patients. Suggested antimicrobial treatment should include Gram-positive and Gramnegative coverage. Given the small amount of published articles, additional studies to validate our conclusions are required. 


\section{References}

1. Glynn M., Rhodes P. Estimated HIV prevalence in the United States at the end of 2003. Natl HIV Prev Conf 2005 June 1215.

2. Centers for Disease Control and Prevention. HIV/AIDS surveillance report, 2005. 2007:10.

3. Rao T.K., Filippone E.J., Nicastri A.D., et al. Associated focal and segmental glomerulosclerosis in the acquired immunodeficiency syndrome. N Engl J Med 1984;310(11):669-73.

4. Gupta S.K., Eustace J.A., Winston J.A., et al. Guidelines for the management of chronic kidney disease in HIV-infected patients: Recommendations of the HIV Medicine Association of the Infectious Diseases Society of America. Clin Infect Dis 2005;40(11):1559-85.

5. Rao T.K. Human immunodeficiency virus infection in end-stage renal disease patients. Semin Dial $2003 ; 16(3): 233-44$.

6. Ahuja T.S., Grady J., Khan S. Changing trends in the survival of dialysis patients with human immunodeficiency virus in the United States. J Am Soc Nephrol 2002;13(7):1889-93.

7. National Institute of Diabetes and Digestive Kidney Diseases. United States Renal Data System: USRDS 1997 Annual data report. Bethesda, MD: NIH publication; 1997. Report No.: 92-3176.

8. Palella F.J.,Jr., Delaney K.M., Moorman A.C., et al. Declining morbidity and mortality among patients with advanced human immunodeficiency virus infection. HIV Outpatient Study investigators. N Engl J Med 1998;338(13):853-60.

9. Sackoff J.E., Hanna D.B., Pfeiffer M.R., Torian L.V. Causes of death among persons with AIDS in the era of highly active antiretroviral therapy: New York City. Ann Intern Med 2006;145(6):397-406.

10. Manian F.A. Vascular and cardiac infections in end-stage renal disease. Am J Med Sci 2003;325(4):243-50.

11. III. NKF-K/DOQI clinical practice guidelines for vascular access: Update 2000. Am J Kidney Dis 2001;37(1 Suppl 1):S137-81.

12. Mandayam S., Ahuja T.S. Dialyzing a patient with human immunodeficiency virus infection: What a nephrologist needs to know. Am J Nephrol 2004;24(5):511-21.

13. de Silva T.I., Post F.A., Griffin M.D., Dockrell D.H. HIV-1 infection and the kidney: An evolving challenge in HIV medicine. Mayo Clin Proc 2007;82(9):1103-16.

14. Nannery W.M., Stoldt H.S., Fares L.G., 2nd. Hemodialysis access operations performed upon patients with human immunodeficiency virus. Surg Gynecol Obstet 1991;173(5):387-90.

15. Brock J.S., Sussman M., Wamsley M., et al. The influence of human immunodeficiency virus infection and intravenous drug abuse on complications of hemodialysis access surgery. J Vasc Surg 1992;16(6):904,10;911-2.

16. Curi M.A., Pappas P.J., Silva M.B.,Jr., et al. Hemodialysis access: Influence of the human immunodeficiency virus on patency and infection rates. J Vasc Surg 1999;29(4):608-16.

17. Mokrzycki M.H., Schroppel B., von Gersdorff G., et al. Tunneledcuffed catheter associated infections in hemodialysis patients who are seropositive for the human immunodeficiency virus. J Am Soc Nephrol 2000;11(11):2122-7.

18. Gorski T.F., Gorski Y.C., Muney J. Complications of hemodialysis access in HIV-positive patients. Am Surg 2002;68(12):1104-6.

19. Eustace J.A., Gregory P.C., Krishnan M., et al. Influence of intravenous drug abuse on vascular access placement and survival in HIV-seropositive patients. Nephron Clin Pract 2005;100(2):c38-45

20. Mitchell D., Krishnasami Z., Allon M. Catheter-related bacteraemia in haemodialysis patients with HIV infection. Nephrol Dial Transplant 2006;21(11):3185-8.

21. Mitchell D., Krishnasami Z., Young C.J., Allon M. Arteriovenous access outcomes in haemodialysis patients with HIV infection. Nephrol Dial Transplant 2007;22(2):465-70.

22. Moran G.J., Krishnadasan A., Gorwitz R.J., et al. Methicillinresistant $S$. aureus infections among patients in the emergency department. N Engl J Med 2006;355(7):666-74.

23. Klevens R.M., Morrison M.A., Nadle J., et al. Invasive methicillinresistant Staphylococcus aureus infections in the united states. JAMA 2007;298(15):1763-71.

24. Diep B.A., Chambers H.F., Graber C.J., et al. Emergence of multidrug-resistant, community-associated, methicillin-resistant Staphylococcus aureus clone USA300 in men who have sex with men. Ann Intern Med 2008;148(4):249-57. 